\title{
MedienPädagogik
}

www. medienpaed.com

Zeitschrift für

Theorie und Praxis

der Medienbildung

ISSN 1424-3636

\section{Die Wirksamkeit von Medienbildungsinitiativen: Erfolge, Probleme und Lösungsansätze}

\author{
Eine Einschätzung der Schulleitungen und Lehrkräfte \\ allgemeinbildender Schulen der Stadt Frankfurt am Main
}

Yvonne Ludewig, Thomas Knaus und Nicola Döring

\begin{abstract}
Neben der Persönlichkeitsbildung obliegt der Schule ein Qualifizierungsauftrag (KMK 2012, 3). Im Zusammenhang dieses Auftrags, Schülerinnen und Schüler auf das (Berufs-) Leben vorzubereiten, hielten digitale Medien Einzug in nahezu alle Schulformen. Dort sollen sie neben der Förderung der Medienkompetenz in allen ihren Ausprägungen auf Seiten der Schülerinnen und Schüler, zudem die Lehr- und Lernkultur verbessern. Mit Hilfe landes- und städteweiter Initiativen wird dabei die Medienintegration und konkrete Mediennutzung vorangetrieben, wie bspw. durch die Medienbildungsinitiative der Stadt Frankfurt am Main, deren Erfolge, Probleme und Lösungsansätze nach zehnjährigem Bestehen in diesem Artikel betrachtet werden.

Ziel war es dabei, den aktuellen Entwicklungsstand, noch bestehende Probleme und vor allem deren mögliche Lösung aus Perspektive der Lehrkräfte darzustellen. Insgesamt wurden Mängel bei der IT-Infrastruktur, den mediendidaktischen Unterstützungsangeboten und hinderliche organisatorische Rahmenbedingungen identifiziert.
\end{abstract}

\section{Einleitung}

Mit Hilfe landes- und städteweiter Initiativen wird in Deutschland die Integration digitaler Medien in Lehr- und Lernprozesse an Schulen vorangetrieben. Die Medienbildungsinitiative der Stadt Frankfurt am Main, die seit über zehn Jahren besteht, ist hierfür ein Beispiel. Der vorliegende Beitrag beschreibt exemplarisch, wie Schulleitungen und Lehrkräfte an allgemeinbildenden Schulen in Frankfurt am Main den Einsatz digitaler Medien im Unterricht erleben und bewerten. Es wird den Fragen nachgegangen, wie sich schulische Integration und die konkrete Nutzung digitaler Medien im Unterricht nach zehnjähriger Initiative der Stadt Frankfurt am Main und der beteiligten Institutionen veränderte. Welche Verbesserungen konnten erzielt werden und welche Probleme bestehen bis heute? Mit welchen 
Schwierigkeiten wurden die Akteurinnen und Akteure konfrontiert und welche Lösungsansätze konnten sie entwickeln?

Die vorgestellten Ergebnisse wurden im Rahmen einer umfangreicheren Evaluationsstudie gewonnen, welche Rahmenbedingungen und Verbesserungsmöglichkeiten des Kooperationsprojekts «fraLine» der Stadt Frankfurt am Main und des Frankfurter Technologiezentrums [:Medien] an der Fachhochschule Frankfurt am Main untersuchte und dabei Schulleitungen, Lehrkräfte und IT-Beauftragte als zentrale Anspruchsgruppen einbezog.

Im Folgenden wird zunächst die Bedeutung des Einsatzes digitaler Medien an Schulen skizziert und die Medienbildungsinitiative der Stadt Frankfurt am Main vorgestellt. Anschliessend wird auf der Basis von Experteninterviews mit 21 Schulleitungen, einer Befragung von 194 Lehrkräften allgemeinbildender Schulen und drei Fokusgruppen-Diskussionen mit insgesamt 16 Lehrkräften dargestellt, wie die Befragten die jüngsten Entwicklungen bezüglich des schulischen Medieneinsatzes erleben, mit welchen Hemmnissen und Problemen sie konfrontiert sind und welche Lösungsansätze sie vorschlagen.

\section{Relevanz von Medienbildungsinitiativen für den Einsatz digitaler Medien in der Schule}

Der Einsatz digitaler Medien im Schulunterricht verfolgt im Wesentlichen zwei Zielsetzungen, die im Rahmen von Medienbildungsinitiativen umgesetzt werden sollen:

1. die Ausbildung von Medienkompetenz auf Seiten der Schülerinnen und Schüler (vgl. Knaus 2011, 33f.) und

2. die didaktische Weiterentwicklung des Fachunterrichts (vgl. Knaus 2011, 24f.).

\section{Ausbildung von Medienkompetenz auf Seiten der Schülerinnen und Schüler}

Die erstgenannte Zielsetzung begründet sich vor allem aus der Omnipräsenz digitaler Medien: Die digitalen Medien sind im Alltag von Kindern und Jugendlichen in Deutschland fest verankert. So nutzen laut ARD/ZDF-Online-Studie heute alle Jugendlichen (100 Prozent) in Deutschland das Internet zumindest gelegentlich (vgl. Eimeren u. Frees 2012, 363).

Intensive Mediennutzung ist jedoch nicht gleichzusetzen mit kompetenter Mediennutzung: Computer bzw. Tablet, Internet und Smartphone zielgerichtet, sachgerecht, selbstbestimmt und kreativ sowie gleichzeitig selbst- und sozialverantwortlich nutzen zu können, setzt eine Reihe von jeweils medienspezifischen Kenntnissen voraus (vgl. Tulodziecki 1998, 697 ff.). Dazu gehören auch das Wissen und die Fähigkeit, medial vermittelte Informationen zu entschlüsseln, herzustellen und kritisch zu reflektieren (vgl. Knaus 2009, 52ff., 211). Medienkompetenz wird bislang in Deutschland vor allem im Elternhaus und unter Gleichaltrigen erworben. 
Dies führt zu messbaren sozialen Ungleichheiten (vgl. u. a. Moser 2010, 146f.). Dementsprechend sind die allgemeinbildenden Schulen als zentrale Vermittlungsinstanzen gefordert, alle Mädchen und Jungen - unabhängig von ihrer sozialen Herkunft - gleichermassen darin zu unterstützen, zeitgemässe Medienkompetenzen zu entwickeln (vgl. Moser 2010, 146 f.; Senkbeil 2005, 99; Tulodziecki 2011). Diese sind notwendige Voraussetzungen für die Partizipation des Individuums in einer durch digitale Medien geprägten Gesellschaft.

\section{Didaktische Weiterentwicklung des Fachunterrichts}

Neben der Förderung der Medienkompetenzentwicklung verbindet sich mit dem Einsatz digitaler Medien im Unterricht zudem die Hoffnung auf didaktische Verbesserungen (vgl. Döring u. Kleeberg 2006; Knaus 2011, 34 f.): Geeignete Lernprogramme können individuelle Lernbedürfnisse besser bedienen als herkömmlicher Unterricht. Online-Dienste wie Google-Earth unterstützen den Geografie-Unterricht anschaulicher als herkömmliches zweidimensionales Kartenmaterial. Onlineangebote verbessern darüber hinaus den Transfer des Erlernten, da sie nicht nur in der Schule, sondern auch zuhause und unterwegs zur Verfügung stehen. In internationalen Online-Foren und Chats können Fremdsprachenkenntnisse unter Realbedingungen erprobt und erweitert werden.

\section{Rahmenbedingungen für den Einsatz digitaler Medien in der Schule}

Ebenso wie die Medienkompetenzförderung setzt auch die didaktische Verbesserung des Unterrichts mittels digitaler Medien entsprechende technische, organisatorische und persönliche Rahmenbedingungen voraus: Dazu gehören eine angemessene schulische IT-Infrastruktur samt adäquater technischer Betreuung (vgl. Engel et al. 2011, 47 ff.), die systematische curriculare Integration der digitalen Medien durch schulische Medienkonzepte sowie mediendidaktische Beratungsund Unterstützungsangebote wie beispielsweise Fortbildungen und Materialien (vgl. Wiggenhorn u. Vorndran 2003, 23-29; 36 ff.; 43; Owston 2007, 72-73; Knaus 2011, 40 f.; Breiter u. Welling 2010, 16). Nicht zuletzt sind medienspezifische neue Qualifikationen auf Seiten der Lehrkräfte notwendig: Angefangen bei der eigenen Medienkompetenz bis zu geeigneten gruppenpädagogischen und methodischdidaktischen Qualifikationen (vgl. Herzig u. Grafe 2007, 181; Knaus 2009, 216; Niesyto 2009, 15).

\section{Die Medienbildungsinitiative der Stadt Frankfurt am Main}

Die Medienbildungsinitiative der Stadt Frankfurt am Main hat in den letzten zehn Jahren die notwendigen Rahmenbedingungen für den Medieneinsatz im Unterricht geschaffen: 


\section{IT-Infrastruktur und deren technische Betreuung}

Im Zeitraum von 2001 bis 2011 wurden die 152 allgemeinbildenden Frankfurter Schulen auf Basis eigens entwickelter (technischer) Medienkonzepte mit der nötigen IT-Infrastruktur - Hardware, Software und Vernetzung - und dem dafür notwendigen technischen Support vom Stadtschulamt der Stadt Frankfurt am Main ausgestattet. Unterstützt wurde es dabei durch den Caritasverband und dem Amt für Informations- und Kommunikationstechnik. Den technischen Support realisierten (und realisieren bis heute) Studierende von «fraLine», einem Kooperationsprojekt der Stadt Frankfurt und dem FTzM an der Fachhochschule Frankfurt am Main. Zur besseren Koordination und Kommunikation, denn in Frankfurt arbeiten knapp 6000 Lehrkräfte, wurden an allen beteiligten Schulen so genannte «IT-Beauftragte» ernannt, die sich innerhalb ihrer Schule um die Meldung von Störungen oder neuen Anforderungen an die IT-Infrastruktur kümmern. Hierfür wurden die IT-Beauftragten durch das Team des FTzM insbesondere durch technische Basisschulungen und Fortbildungsangebote zu schulspezifischen (medien-) technischen Themen fortgebildet (vgl. www.fraline.de, aufgerufen am 1. Nov. 2012).

\section{Mediendidaktische Beratungs- und Unterstützungsangebote}

Mediendidaktische Unterstützung erhalten die Frankfurter Schulen vom Staatlichen Schulamt für die Stadt Frankfurt am Main und weiteren Institutionen (vgl. www.medienbildung-frankfurt.de) in Form von Fortbildungen. Daneben können Grund- und Förderschulen auf zusätzliche Unterstützung durch Fachberaterinnen und Fachberater sowie Fachgruppen zurückgreifen. Diese helfen bei Abstimmungen des Softwareeinsatzes und der Suche passender Fortbildungen. Darüber hinaus geben sie Hilfestellungen für den praktischen Einsatz der digitalen Medien im Unterricht. Die Förderschulen verfügen über eine schulübergreifende Arbeitsgruppe, die sich mit dem Einsatz digitaler Medien im Unterricht befasst und dabei gemeinsam Ideen und Einsatzmöglichkeiten entwickelt. Zusätzlich engagieren sich die Forschenden, Studierenden und Mitarbeiterinnen und Mitarbeiter der Fachhochschule Frankfurt in Kooperation mit dem Staatlichen Schulamt im Bereich der Medienbildung (Engel et al. 2010, 45ff.): So werden speziell auf die örtlichen Begebenheiten zugeschnittene Seminare für Lehrkräfte organisiert und das niederschwellige Projekt «Unterrichtsbegleitung» (vgl. www.unterrichtsbegleitung. de, aufgerufen am 16. Jul. 2013) angeboten. Mediendidaktische Unterstützung erhalten die Lehrkräfte darüber hinaus auch durch das Frankfurter Medienzentrum, welches neben Fortbildungen auch ein umfassendes Mediensortiment anbietet. 


\section{Ergebnisse bisheriger Evaluationen der Medienbildungsinitiative}

Zur stetigen Weiterentwicklung der Medienbildungsinitiative wurden bereits zwei Evaluationen im Jahre 2003 und 2007 durch das Institut für Informationsmanagement Bremen GmbH (ifib) durchgeführt (siehe Wiedwald et al. 2003; Wiedwald et al. 2007). Dabei zeigten sich insbesondere Mängel an der IT-Infrastruktur, den medienpädagogischen Konzepten der Schulen und der medienpädagogischen Unterstützung (ebd. 2007, 27). Basierend auf diesen Erkenntnissen wurden Verbesserungen in Bezug auf die IT-Ausstattung vorgenommen und Weiterentwicklungen im Bereich der medienpädagogischen Unterstützungsangebote angestrebt - u. a. entstand daraus das Angebot der «Unterrichtsbegleitung» (vgl. Thülen 2009, 142f.).

Neben der Erfassung der Auffassungen der Lehrkräfte hinsichtlich des bisher Realisierten zielt die in diesem Beitrag vorgestellte Evaluation darauf ab, bestehende Probleme zu identifizieren und damit die Weiterentwicklung der Medienbildungsinitiative durch adäquate Lösungsansätze zu unterstützen. Im Mittelpunkt der Untersuchung standen dabei folgende Fragestellungen:

Frage 1: Wie ist die aktuelle Mediensituation an den allgemeinbildenden Frankfurter Schulen und wie erleben Lehrkräfte den Einsatz digitaler Medien im Unterricht? Frage 2: Mit welchen Hemmnissen und Problemen beim Einsatz digitaler Medien im Unterricht sind Lehrkräfte konfrontiert?

Frage 3: Welche Lösungsansätze zur Erleichterung des Einsatzes digitaler Medien im Unterricht schlagen Lehrkräfte vor?

\section{Methodisches Vorgehen}

Die summativen (aktuelle Mediensituation) und formativen (Probleme und Lösungsansätze) Evaluationselemente wurden mittels Mixed-Methods-Design bestehend aus 21 Experteninterviews mit Schulleitungen, einer Paper-Pencil-Befragung und drei Fokusgruppen-Diskussionen mit Lehrkräften untersucht. Dafür wurde zu Beginn eine Stichprobe von $n=21$ Schulen aus der Grundgesamtheit der allgemeinbildenden Schulen Frankfurts $(n=152)$ proportional anhand einer Zufallsziehung via Quotenplan gezogen (siehe Tabelle 1).

Die Teilnahme an den Experteninterviews, Fragebogenerhebungen und Fokusgruppen-Diskussionen basierte jeweils auf Freiwilligkeit. Daher liegt keine statistische Repräsentativität der Stichprobendaten vor. Eine Vollerhebung aller 152 Frankfurter Schulen bzw. der dort tätigen rund 5.600 Lehrkräfte (darunter ca. 600 IT-Beauftragte und 300 Schulleitungen) war aus forschungsökonomischen Gründen nicht möglich. Bei entsprechenden Selbstselektions-Stichproben ist typischerweise davon auszugehen, dass besonders engagierte und interessierte Personen tendenziell überrepräsentiert sind. Dadurch ergibt sich das Problem, dass wahr- 
scheinlich die Medienintegration in durchschnittlichen und unterdurchschnittlichen Schulen in geringerem Masse berücksichtigt wurde.

\section{Überblick der Stichproben}

\begin{tabular}{|l|l|l|l|}
\hline Schulform & Schulen Population & Schulen Stichprobe & $\begin{array}{l}\text { Lehrkräfte } \\
\text { Stichprobe* }\end{array}$ \\
\hline Grundschulen & 77 & 11 & 85 \\
\hline Förderschulen & 14 & 2 & 31 \\
\hline Hauptschulen & 9 & 1 & 12 \\
\hline Realschulen & 16 & 2 & 16 \\
\hline Gesamtschulen & 13 & 2 & 21 \\
\hline Gymnasien & 21 & 3 & 29 \\
\hline $\mathrm{n}$ & 152 & 21 & 194 \\
\hline
\end{tabular}

Tabelle 1: Zufallsstichprobe und Quotenverteilung der allgemeinbildenden Schulen (Stand: März 2010)

(*) Die Stichprobe der Lehrkräfte basiert auf der Stichprobe der Schulen $(n=21)$

Anhand der Experteninterviews mit den Schulleitungen wurde zu Beginn ein Überblick über die aktuelle Mediensituation (Ausstattungssituation und Mediennutzung) der Schulen erfasst. Ein halbstandardisierter Interview-Leitfaden bildete die Basis relevanter Fragestellungen. Vorteil des halbstandardisierten Experteninterviews war, dass im Falle weiterführender Fragen vom Leitfaden abgewichen werden konnte (vgl. Bortz u. Döring 2006, 239). Die Experteninterviews wurden im März 2010 mit den 21 Schulleitungen der zuvor ausgewählten allgemeinbildenden Schulen durchgeführt und dauerten zwischen 30 und 45 Minuten. Sie wurden aufgezeichnet, vollständig verschriftlicht und mit der Methode der qualitativen Inhaltsanalyse ausgewertet. Die Ergebnisse der Experteninterviews dienten zudem als Basis für die Entwicklung der Paper-Pencil-Befragung der Lehrkräfte, welche anschliessend konzipiert, einem Pretest unterzogen und durchgeführt wurde. Die Befragung erfolgte ebenfalls an den 21 ausgewählten Schulen und erfasste die Mediensituation (Ausstattungssituation und Mediennutzung) und die Einstellung der Lehrkräfte gegenüber dem Einsatz digitaler Medien im Unterricht. Die Fragebögen wurden den Schulleitungen nach den Experteninterviews übergeben und im Rahmen von Lehrerkonferenzen oder vergleichbaren Zusammenkünften ausgefüllt. Insgesamt nahmen 194 Lehrkräfte an der Befragung teil. Die Fragebögen wurden in die Datenmatrix des Statistikprogramms SPSS übertragen und quantitativ ausgewertet. 
Abschliessend wurden drei Fokusgruppen-Diskussionen durchgeführt. Anhand eines Diskussionsleitfadens wurden dabei ausgewählte Themen, die sich im Rahmen der Experteninterviews und der Paper-Pencil-Befragung als besonders relevant herausstellten, vertieft und diskutiert. Die Fokusgruppen-Diskussionen wurden im Mai 2010 in drei Frankfurter Schulen durchgeführt und dauerten jeweils zwischen 30 und 45 Minuten. Sie wurden ebenfalls aufgezeichnet, vollständig verschriftlicht und mit der Methode der qualitativen Inhaltsanalyse ausgewertet. Insgesamt nahmen 16 Lehrkräfte an den Fokusgruppen-Diskussionen teil (sechs Lehrkräfte einer Grundschule, fünf Lehrkräfte einer Förderschule und fünf Lehrkräfte eines Gymnasiums).

\section{Ergebnisse}

Die Schulleitungen und Lehrkräfte stehen dem Einsatz digitaler Medien in Schule und Unterricht positiv gegenüber und berichteten über Erfolgserlebnisse. Wie die im Folgenden aufgeführten Beispiele und Zitate zeigen, bestehen jedoch noch Mängel hinsichtlich der technischen und organisatorischen Rahmenbedingungen.

Frage 1: Wie ist die aktuelle Mediensituation (im Wesentlichen in Bezug auf die sächliche Ausstattung mit digitalen Medien und deren Nutzung) an den allgemeinbildenden Frankfurter Schulen und wie erleben Lehrkräfte den Einsatz digitaler Medien im Unterricht?

Die Befragten berichteten über eine gute bis befriedigende Medienausstattung in den Schulen, die aber weiter verbesserungswürdig ist. Zudem wird über positive Erfahrungen mit dem Einsatz digitaler Medien im Unterricht berichtet. Die wechselseitige Unterstützung im Kollegenkreis spielte bei der Integration von Medien eine wesentliche Rolle; externe Angebote wurden indessen zurückhaltender genutzt.

\section{Gute bis ausreichende Medienausstattung in den Schulen}

Zum Zeitpunkt der Befragung (2010) verfügten alle allgemeinbildenden Schulen Frankfurts, die an der Untersuchung teilnahmen, ausgenommen einer Grundschule (aufgrund fehlender Räumlichkeiten), über mindestens einen ComputerFachraum. Daneben besassen, ausser den Gymnasien, alle Schulen in Fach- oder Klassenräumen «Medien-Ecken» mit jeweils zwei bis vier PCs. Nur wenige Schulen verfügten über einen Notebooksatz mit 13 bis 15 Notebooks (jeweils eine Grund-, Haupt-, Real- und Gesamtschule) oder digitale Tafeln (drei Gymnasien und zwei Grundschulen). 


\section{Positive Erfahrungen mit dem Medieneinsatz}

Insgesamt standen alle befragten Lehrkräfte aus allgemeinbildenden Schulen in Frankfurt am Main den digitalen Medien positiv gegenüber und nutzten diese häufig im Unterricht und zur Vor- und Nachbereitung der Unterrichtsstunden. Dabei war die Mehrheit (80\%) mit den digitalen Medien ihrer Schule vertraut. Nur wenige kannten sich geringfügig (11\%) oder gar nicht (9\%) aus. Die innerhalb der Gruppen-Diskussion befragten Lehrkräfte der Grundschule und des Gymnasiums setzten etwa zwei bis drei Mal pro Woche und die Lehrkräfte der Förderschule täglich digitale Medien im Unterricht ein. Die Mediennutzung im Schulunterricht umfasste dabei häufig das Einüben grundlegender Bedienschritte, den Einsatz von Office- und Lernprogrammen und das Recherchieren im Internet.

Die befragten Lehrkräfte erlebten bei ihrer Mediennutzung vorwiegend die Vorteile der digitalen Medien. So liessen sich manche Sachverhalte verständlicher und einfacher darstellen (84\%), der Unterricht konnte «interaktiver» gestaltet und die Schülerinnen und Schüler konnten «leichter motiviert werden» (69\%). Lehrerinnen und Lehrer konstatierten, dass ihre Schülerinnen und Schüler beim Einsatz digitaler Medien im Unterricht interessierter und aufmerksamer wären. Ob diese Motivationssteigerung struktureller oder vorübergehender Natur sind, konnte im Rahmen der Diskussionen aber nicht zweifelsfrei geklärt werden. Zudem war die Vor- und Nachbereitung der Unterrichtsstunden durch die Zuhilfenahme der digitalen Medien einfacher und schneller zu realisieren, so dass die Lehrkräfte nach dem anfänglichen Initialaufwand - weniger Zeit benötigten und sich entlastet fühlten (85\%). 16\% der Lehrkräfte thematisierten diesen Initialaufwand, der für die Auseinandersetzung und sinnvolle didaktische Integration digitaler Medien im Unterricht nötig sei und der ihrer Meinung nach abschreckend auf andere Lehrkräfte wirken könne.

\section{Interne und externe Unterstützungsangebote}

Bei Fragen und Problemen hinsichtlich des Einsatzes digitaler Medien im Unterricht griffen die Lehrkräfte in erster Linie auf die Unterstützung erfahrener Kolleginnen und Kollegen der eigenen Schule zurück (68\%) oder nahmen an den schulinternen Fortbildungen teil (45\%). Hilfen und Unterstützungen externer Anbieter, wie bspw. die Fortbildungen vom Amt für Lehrerbildung (27\%), dem Medienzentrum Frankfurt e.V. (29\%) oder der Unterrichtsbegleitung vom FTzM (9\%), wurden hingegen seltener wahrgenommen. Die genauere Betrachtung zeigt, dass die geringere Nutzung auf die geringe Bekanntheit der Angebote zurückgeführt werden kann. So war bspw. nur $43 \%$ der 194 befragten Lehrkräfte die Unterrichtsbegleitung bekannt. Nur 15\% der befragten Lehrkräfte hatten bereits Gelegenheit gehabt, die Unterrichtsbegleitung zu nutzen, ein niederschwelliges und individualisiertes Angebot (vgl. Thülen 2009, 142f.), welches insgesamt sehr positiv bewertet wird: Die 
befragten Lehrkräfte, die das Angebot bereits nutzten, waren vorwiegend damit zufrieden $(M=1,7 ; S D=0,83$; auf einer Skala von $1=$ sehr zufrieden bis $6=$ sehr unzufrieden). Auch 4 der 16 Fokusgruppenteilnehmer/innen bewerteten die Unterrichtsbegleitung als sehr hilfreich.

Neben der Unterrichtsbegleitung verantwortet das Team des FTzM vor allem den technischen Support der IT-Infrastruktur der Frankfurter Schulen («fraLine»). Dieser Support wurde von allen befragten Lehrkräften als schnell und kompetent gelobt. Gleichzeitig wurde aber auch deutlich, dass trotz des als hilfreich wahrgenommenen fraLine-Supports der Einsatz digitaler Medien im Unterricht auf diverse Hemmnisse stösst.

Frage 2: Mit welchen Hemmnissen und Problemen beim Einsatz digitaler Medien im Unterricht sind Lehrkräfte konfrontiert?

Insgesamt 66\% der 194 per Fragebogen befragten Lehrkräfte wünschten sich eine zuverlässiger einsetzbare technische Ausstattung. Auch das Fehlen konkreter Unterrichtskonzepte wurde als Hemmnis eines breiteren Einsatzes digitaler Medien im Unterricht diskutiert. Dies wiederum wurde unter anderem auf das Fehlen notwendiger zeitlicher und finanzieller Ressourcen zurückgeführt sowie auf Mängel bei den bestehenden Weiterbildungsangeboten.

\section{Veraltete Computertechnik:}

In den Fokusgruppen-Diskussionen zeigten sich nur die Förderschullehrkräfte sehr zufrieden mit ihrer technischen Ausstattung (PC-Raum, PC-Ecken in allen anderen Schulräumen und mobile Präsentationseinheiten). Die Lehrkräfte der anderen Schultypen waren weniger zufrieden. Schwierigkeiten durch technische Probleme oder fehlende Hard- und Software führten laut der befragten Lehrkräfte bereits dazu, dass der geplante Medieneinsatz nur beschränkt oder teilweise gar nicht stattfinden konnte. Dies verursachte insbesondere bei Grundschullehrkräften nicht selten Motivationsverluste und schreckte gerade ungeübtere Lehrkräfte ab:

Das ist eigentlich das Hauptproblem, weil die Computer auch einfach uralt sind. Und die stürzen bei vielen Lernprogrammen auch regelmässig ab. Also es ist richtig anstrengend [...] Es ist immer irgendetwas, was das erschwert und sicherlich einigen Kollegen das Ganze auch noch einmal weniger schmackhaft macht. (Lehrerin, Grundschule) 


\section{Fehlende Computerarbeitsplätze in den Klassenräumen:}

Die Gymnasiallehrkräfte hingegen beklagten, dass nicht in jedem Klassenraum genügend Computerarbeitsplätze für die Schüler/innen zur Verfügung stehen und somit meist nur Lehrkräfte die digitalen Medien im Unterricht nutzen:

Im Moment sind wir dankbar, dass wir zwar lehrerzentriert, aber trotzdem die Möglichkeiten des Beamers, Notebooks und auch des Internetzugangs nutzen können. (Lehrer, Gymnasium)

Der Beamer allein nützt noch nichts [...]. Die Nutzbarkeit für die Schüler wird halt erst dann möglich, wenn [es] wirklich acht, zehn Rechner [gibt] - die man auch verteilen kann und spontan sagt: 〈Jetzt recherchiert mal in Zweiergruppen». (Lehrer, Gymnasium)

\section{Fehlende konkrete Unterrichtskonzepte:}

Neben den technischen Schwierigkeiten stiessen einige der befragten Lehrkräfte auf Probleme beim methodisch-didaktischen Einsatz der digitalen Medien (59\%). Dieser wurde zum Zeitpunkt der Befragung nur an 12 der 21 allgemeinbildenden Schulen Frankfurts im Schulprogramm mittels eines Medienkonzepts festgelegt. Die Fokusgruppenteilnehmer/innen berichteten in diesem Zusammenhang über ihre Schwierigkeiten, für den eigenen Fachunterricht passende Unterrichtskonzepte zu entwickeln und umzusetzen:

Wie kann ich das moderne Medium mit seinen neuesten Möglichkeiten nutzen? Das, was alles momentan auf einen einströmt - von wegen YouTube, Twitter und was es da alles gibt - dass man das so einbinden kann und damit relativ schnell Ergebnisse erzielen kann, das ist im Moment einfach nicht so ganz klar. (Lehrer, Gymnasium)

Fehlende zeitliche und finanzielle Ressourcen, um Unterrichtkonzepte selbst zu entwickeln oder Fortbildungen wahrzunehmen:

Insbesondere Grundschullehrkräfte nannten den Mangel an freier Zeit als Problem bei der Umsetzung ihrer Ideen in konkrete Unterrichtskonzepte. Auch Gymnasiallehrkräfte bemängelten die geringe Zeit, die ihnen für Fortbildungen zur Verfügung steht, und kritisierten gleichzeitig die fehlenden finanziellen Ressourcen der Schulen für kostenpflichtige Fortbildungen:

Lehrer haben schon [...] immer wieder neue Ideen. Aber es ist immer das Zeitproblem und die Umsetzung. (Lehrerin, Grundschule) 
Die Schulen sind finanziell ziemlich knapp [...] an der eigenen Schule läuft eine Menge Kompetenz rum und [...] wenn ich ein Angebot wahrnehme, wofür ich bezahlen muss, dann will ich das nicht. Nicht, weil ich das nicht für Nichts wert halte, einfach weil kein Geld da ist - für Nichts. (Lehrer, Gymnasium)

\section{Mängel bei den Weiterbildungsangeboten:}

Bei besuchten Weiterbildungsangeboten wurden vereinzelt zu geringe Praxisnähe und mangelnde Transfermöglichkeiten beklagt. So können die Inhalte externer Schulungen selten auf den eigenen Unterricht übertragen werden, wenn vor Ort nicht die notwendige bzw. identische Hard- und Software zur Verfügung steht:

Es [wird gesagt] [...] was man alles machen kann und wie schön [es funktioniert]. Aber wenn man dann am nächsten Tag in die Schule kommt, sieht alles ganz anders aus und dann hört man gleich wieder auf. So geht es einem ja oft nach Fortbildungen. (Lehrerin, Grundschule)

Die Fortbildungsstruktur in Hessen, was die Lehrerbildung anbetrifft, ist da immer hinterher hinkend gewesen. [...] Jetzt will kein Mensch mehr wissen, wie ein Beamer funktioniert [...] Sondern, wie setze ich das in meinem Fach ein, gibt es da schon ein Beispiel der Nutzung? Und die fachbezogenen Anwendungen von neuen Medien sind zu wenig, viel zu wenig. (Lehrer, Gymnasium)

Die Lehrkräfte bemerken ihre eigenen Wissensdefizite hinsichtlich ihrer Kompetenz, die technischen Geräte zu bedienen:

Also gerade [...] Beamer und Computer koppeln. Also da hätte ich momentan einfach viel zu sehr Angst [...]. Da bin ich nicht fit genug. (Lehrer, Förderschule)

Also ich fand es auch traurig, dass wir hier dann auch überall Beamer hängen hatten und keiner wusste, wie er sie bedienen sollte. (Lehrer, Gymnasium)

Gleichzeitig wurden Schulungen zu technischen Themen von der Lehrerschaft wenig angenommen und teilweise als zu kompliziert kritisiert:

Ich glaube nicht [...], dass [technische Basisschulungen] gross Anklang finden würden. Für die IT-Leute gibt es solche Seminare [...] Aber ganz ehrlich - ich fand es ganz schön komplex teilweise. Und ich bin IT-Beauftragte und 
da quäl ich mich da durch. Das ruft kein Interesse hervor. (Lehrerin, Grundschule)

Zur Überwindung der zuvor genannten Probleme schlugen die Teilnehmerinnen und Teilnehmer der Experteninterviews, Paper-Pencil-Befragung und Fokusgruppen folgende Lösungsansätze vor:

Verbesserung der IT-Infrastruktur an den Schulen:

Besonders in den Grundschulen sollte die vorhandene Hardware und Peripherie erneuert werden, da langsame und fehleranfällige Computertechnik den Medieneinsatz im Unterricht vielerorts noch blockiert. Darüber hinaus sollten mehr Laptops oder Tablet-PCs an Schulen zur Verfügung gestellt werden, um den Medieneinsatz zeitlich und räumlich flexibler gestalten zu können.

\section{Etablierung eines schuleigenen IT-Teams:}

Für den problemlosen Ablauf des Medieneinsatzes ist eine funktionierende IT-Infrastruktur entscheidend. Für die dafür notwendige Wartung und Koordination des zentralen Supports ist die Einrichtung eines schulinternen «IT-Teams» (mindestens bestehend aus bis zu drei Lehrkräften) notwendig, welches mit entsprechenden Entlastungsstunden ausgestattet werden sollte.

\section{Coaching zur Entwicklung eines medienpädagogischen Medienkonzepts für Schulleitungen:}

Zur Unterstützung bei der Entwicklung eines Mediencurriculums bzw. Medienkonzepts bezüglich des didaktischen Einsatzes der digitalen Medien im (Fach-) Unterricht kann ein «medienpädagogisches Coaching» wertvolle Dienste leisten.

\section{Verbreitung von Best-Practice-Beispielen:}

Es sollten Veranstaltungen an interessierten Schulen durchgeführt werden, in denen bewährte Praxisbeispiele («Best Practices») anderer Schulen zum Einsatz digitaler Medien im Unterricht durch fraLine-Mitarbeiterinnen und Mitarbeiter vorgestellt werden. Da das fraLine-Team bereits über umfangreiche Erfahrungen durch regelmässige Besuche an zahlreichen Frankfurter Schulen verfügt, kann es gut bewährte Unterrichtskonzepte sammeln, aufbereiten und in den anderen interessierten Schulen präsentieren. Dadurch erhalten die Lehrkräfte Anregungen und Ideen, wie sie digitale Medien in ihrem Unterricht einsetzen können. fraLine fungiert dabei als Multiplikator, der - die ausdrückliche Genehmigung der Beteiligten vorausgesetzt - Informationen zwischen den Schulen weiterleitet. Anschauliche und praxisnahe Best-Practice-Beispiele könnten zudem als Videoclips (oder anderweitig medial aufbereitet) über eine Webseite bereitgestellt werden. 


\section{Medienpädagogische Seminare für Lehrkräfte:}

Für Lehrkräfte sollten bedarfsgerechte Seminare angeboten werden, welche nicht nur das reine Erlernen der Bedienung digitaler Medien beinhalten, sondern auch deren konkrete Einsatzmöglichkeiten im Unterricht möglichst praxisnah thematisieren. Die Unterrichtskonzepte sollten dabei im Mittelpunkt der Seminare stehen, die notwendigen technischen Kompetenzen lediglich am Rande mitbehandelt werden. Die Seminare sollten nach Möglichkeit vor Ort in den Schulen stattfinden und die lokalen technischen und räumlichen Bedingungen einbeziehen, um die zukünftige Anwendbarkeit des Gelernten zu gewährleisten.

\section{Bereitschaft zum gegenseitigen, kollegialen Lernen:}

Neben notwendigen Fortbildungen sind ein regelmässiger Austausch und Kooperationen unter den Lehrkräften und unter den Schulen förderlich, um neue Einsatzmöglichkeiten digitaler Medien kennenzulernen oder weiterzuentwickeln. Die Bereitschaft zum gegenseitigen, kollegialen Lernen (z. B. durch Hospitationen oder Coachings) sowie das Experimentieren mit den didaktischen Möglichkeiten digitaler Medien sollte auf Seiten der Lehrkräfte bestärkt werden.

\section{Entwicklungsbedarfe}

Trotz messbarer Erfolge und Fortschritte nach zehnjährigen Bemühungen im Rahmen der Medienbildungsinitiative der Stadt Frankfurt am Main wird das volle Potenzial, das digitale Medien für Lehr- und Lernprozesse im schulischen Unterricht bieten könnten, noch nicht genutzt: Die noch immer geringe und einseitige Nutzung von digitalen Lehr- und Lernmedien im Schulalltag ist auf die bestehenden Rahmenbedingungen an den Schulen zurückzuführen, die laut den Befragten noch nicht optimal seien. In diesem Zusammenhang wird besonders über die mangelnde technische Infrastruktur (im Wesentlichen sei dies fehlende und veraltete Hardware) geklagt. Die befragten Lehrkräfte wünschen sich dementsprechend vor allem eine Verbesserung der technischen Infrastruktur ihrer Schulen (bzw. diese dauerhaft auf einem einsatzfähigen Stand zu halten), um eine ausreichende und vor allem stetige Einbindung in den Unterricht zu ermöglichen. Darüber hinaus werden organisatorische (im Wesentlichen fehlende zeitliche und finanzielle Ressourcen) und pädagogische Rahmenbedingungen (fehlende Medienkonzepte und unzureichende medienpädagogisch-technische Qualifizierung der Lehrkräfte) als Probleme angesehen. Eine besondere Herausforderung stellt die medien- und fachdidaktisch versierte Integration der digitalen Medien in den Unterricht dar. Demnach wünschen sich die befragten Lehrkräfte praxisnahe Fortbildungen, in denen konkrete «Best Practice»-Beispiele, wie übertragbare Unterrichtskonzepte, vorgestellt werden. 


\section{Work in progress}

Auf der Grundlage der vorliegenden Ergebnisse wurden in den zurückliegenden eineinhalb Jahren u. a. folgende Aktivitäten im Umfeld der Frankfurter Medienbildungsinitiative angestossen bzw. weiterverfolgt:

- Von Seiten der Stadt Frankfurt wurden die Ausstattungen der Schulen mit neuen, leistungsfähigen Rechnern sukzessive fortgesetzt; aktuell können in den 152 Frankfurter Schulen über 19.243 Rechner im Unterricht eingesetzt werden (vgl. Sorge 2012). Auch die Ausstattung mit digitalen Tafelsystemen wurde um 392 Geräte ergänzt (ebd.);

- Die Stadt Frankfurt setzte in Zusammenarbeit mit den bewährten Kooperationspartnern die Einführung eines «Active-Directory-Standards» sowie die konzeptionelle Entwicklung einer «Schul-Cloud» fort. Damit soll künftig mobiles Lernen verstärkt möglich sein. Die Einführung der Verwaltungsdomäne Active Directory soll das Installations- und Updatemanagement an den 152 Schulen künftig erleichtern. Die konzeptionelle Arbeit zu schulspezifischen Cloudlösungen sollen erweiterte bzw. flexiblere Einsatzmöglichkeiten (z. B. virtualisierte Arbeitsplätze, webbasierte Anwendungs- und Lernsoftware) bieten und gleichzeitig werden mit ihr Hoffnungen zur Entlastung vom kosten- und personalintensiven Betrieb verbunden (vgl. Engel et al. 2012, 49ff.). Unter dem Projekttitel «Schule 2020» sollen die zuvor aufgeführten Teilprojekte weiter verfolgt und unter besonderer Berücksichtigung organisatorischer und finanzieller Gesichtspunkte in ämterübergreifenden Lenkungsrunden beleuchtet werden. Ziel ist die künftige inhaltliche Ausrichtung und organisatorische Anbindung und Ausgestaltung der Frankfurter Medienbildungsinitiative;

- Das Konzept für das im Rahmen der vorliegenden Studie ebenfalls angeregte Projekt «medienpädagogisches Coaching» wurde entwickelt sowie Finanzierungsmöglichkeiten für dieses - zunächst regional begrenzte - Projekt gesucht. Aus Gründen, die im nächsten Abschnitt erläutert werden, wurde die zweite Aufgabe bislang nicht erfüllt;

- Die Inhalte der technischen Grundlagenseminare des FTzM-Teams wurden nach einer genauen Prüfung an die im Rahmen der vorliegenden und einer weiteren Studie (vgl. Knaus u. van Holst 2012, 12f.) erhobenen Bedürfnisse angepasst. Im Jahr 2013 wurden bereits erste Veranstaltungen nach den neu ausgerichteten Inhalten angeboten und im Rahmen der Seminarevaluationen als positiv bewertet;

- Der im Rahmen dieser Studie als Wunsch von Frankfurter Lehrkräften formulierten Bereitstellung «übertragbarer Unterrichtskonzepte» stand das Team des FTzM skeptisch gegenüber, da diese im Unterricht womöglich ohne die nötige umfängliche Beachtung der Ziel- bzw. Lerngruppe und des jeweils zu behandelnden Unterrichtsgegenstands («didaktische Analyse»; vgl. Klafki 1959; 
Klafki 1991, 272) eher unreflektiert angewendet würden. Die Verantwortlichen entschieden sich daher, statt der Weitergabe von «Patentrezepten» für den schulübergreifenden Austausch weiterführender Konzepte und inspirierender Ideen. Zur Intensivierung dieses Austauschs wurde eine regelmässig stattfindende regionale Fachtagung und Medienmesse initiiert (vgl. www.fraMediale. de, aufgerufen am 16. Jul. 2013).

Insgesamt ist festzuhalten, dass die Frankfurter Medienbildungsinitiative auf einem guten Weg des nachhaltigen Einsatzes digitaler Medien in Schulen ist. Insbesondere das Konzept «fraLine», das Kooperationsprojekt der Stadt Frankfurt und des FTzM an der Fachhochschule Frankfurt am Main, durch das Lehrkräfte schnelle, praktische Unterstützung erhalten, die den technischen Support der ITInfrastruktur garantiert, hat sich als wesentlicher Anker innerhalb der regionalen Medienbildung etabliert. Hierdurch werden Lehrkräfte, wie auch von der Kultusministerkonferenz gefordert, «von der technischen Betreuung entlastet, um sich auf die pädagogischen Aufgaben beim Einsatz der digitalen Medien im Unterricht konzentrieren zu können» (KMK 2012, 7 f.).

\section{Ausblick}

Vermutlich sind sowohl die identifizierten Mängel der technischen als auch der organisatorischen Rahmenbedingungen auf den Projektcharakter bzw. das Fehlen einer kontinuierlichen und verlässlichen Perspektive zurückzuführen: Während neue digitale Medien in unserer Lebenswelt eine Konstante bilden (vgl. KMK 2012, $3 \mathrm{f}$.), wird ihnen in Bildungszusammenhängen oft ein «Ausnahmestatus und Eventcharakter» zugewiesen (Knaus 2009, 222f.; Knaus 2011, 30 ff.).

Unterstützungsangebote für die Entwicklung schulischer Medienkonzepte, die die schulische Medienentwicklung und den zielführenden, didaktisch versierten Einsatz digitaler Lehr- und Lernmedien in Unterrichtskontexten fokussieren sowie diesen Medieneinsatz flankierende und bedarfsgerechte Fortbildungsangebote stehen auf der Wunschliste der Schulen ganz oben. Diese Angebote finden jedoch - wenn eine adäquate Finanzierung fehlt - bestenfalls schulintern statt, wenn engagierte Lehrkräfte ihre interessierten Kolleginnen und Kollegen ehrenamtlich (ohne entsprechende Entlastungsstunden) unterstützen. Professionelle und an schulischen Bedürfnissen ausgerichtete Angebote könnten demnach nicht nur den Lehrerinnen und Lehrern zugutekommen, die mit der Integration digitaler Medien in ihren Unterricht Schwierigkeiten haben, sondern kämen auch den «engagierten Medienprofis» unter den Lehrkräften (Gysberts 2008, 168) zugute, da sie diese in ihrer Rolle als interne Fortbildungsanbieterin bzw. interner Fortbildungsanbieter entlasten. 
Als ein wesentliches Grundproblem, nicht nur für die Bereitstellung adäquater Fort- und Weiterbildungsangebote, wird die fehlende (kontinuierliche) Finanzierung benannt. Nur auf Grundlage einer verlässlichen finanziellen Basis kann der teils noch mangelnden IT-Ausstattung und der unzureichenden medienpädagogischen Qualifizierung der Lehrkräfte strukturell und nachhaltig begegnet werden. Eine Schwierigkeit in diesem Zusammenhang sind die unterschiedlichen Zuständigkeiten deutscher Schulen: Während Schulträger für die sächliche Ausstattung von Schulen verantwortlich zeichnen, sind die Kultusministerien für inhaltliche Fragen zuständig und fungieren als Dienstgeber der Lehrerinnen und Lehrer - schulische Medienbildung jedoch, berührt stets sächliche und inhaltliche Fragen.

Im Sinne der Nachhaltigkeit ist es wichtig, Medienbildungsinitiativen immer wieder in ihrer Gesamtheit zu evaluieren und die Ergebnisse zu publizieren, um einen Austausch der verschiedenen Anspruchsgruppen und Akteurinnen und Akteuren zu ermöglichen. Denn künftig sollten nicht nur neue Projekte initiiert, sondern auch an der Nachhaltigkeit bewährter Konzepte gearbeitet werden. Auch die Kultusministerkonferenz fordert in diesem Kontext «eine ganzheitliche, vernetzte Strategie zur nachhaltigen Förderung der Medienbildung in der Schule» (KMK 2012 , 6). Diese Vernetzung kann über einen regen Informationsaustausch über die Hemmnisse und Fortschritte der Initiativen erreicht werden. Denn noch immer verfügt jede Stadt, jeder Kreis, jedes Bundesland über eigene Vorstellungen und voneinander unabhängige Initiativen, die alle zeitgleich der Frage nachgehen, wie Medienbildung in Schule integriert werden kann. Wir verdanken zwar dieser Pluralität zahlreiche gute Ideen, jedoch «erfindet jeder das Rad mehrfach neu», wenn über diese Erkenntnisse nicht in geeigneter Form berichtet wird. Wir berichteten exemplarisch über die Frankfurter Medienbildungsinitiative; nicht, weil wir davon ausgehen, dass das Engagement der Frankfurter besonders vorbildhaft wäre, sondern weil wir überzeugt sind, dass alle Initiativen vom gegenseitigen Austausch über Erfolge, Probleme und Lösungsansätze profitieren können.

\section{Literatur}

Bortz, Jürgen und Nicola Döring. 2006. Forschungsmethoden und Evaluation für Sozial- und Humanwissenschaftler. 4. Auflage. Berlin: Springer.

Breiter, Andreas und Stefan Welling. 2010. «Integration digitaler Medien in den Schulalltag als Mehrebenenproblem.» In Bildung und Schule auf dem Weg in die Wissensgesellschaft, hrsg. v. Birgit Eickelmann, 13-25. Münster: Waxmann.

Döring, Nicola und Nicole Kleeberg. 2006. «Mobiles Lernen in der Schule - Entwicklungs- und Forschungsstand.» Unterrichtswissenschaft - Zeitschrift für Lernforschung 34 (1): 70-92. 
Engel, Olga, Thomas Knaus, Anthony Schmelz und Ulrich Schrader. 2010. «fraLine IT-Schul-Service: Ein Kooperationsprojekt der Stadt Frankfurt am Main - Stadtschulamt und der Fachhochschule Frankfurt am Main - University of Applied Sciences.» Dritter Bericht in der 3. Projektlaufzeit von September 2007 bis August 2011. http://www.fraline.de/images/stories/fraLineData/publikationen/ projektberichte/zwischenbericht_fraLine_dritte_projektlaufzeit_august_2010. pdf [aufgerufen am 16.7.2013].

Engel, Olga, Thomas Knaus, Anthony Schmelz und Thülen Katharina. 2012. «Projekt fraLine (4): Ein Kooperationsprojekt der Stadt Frankfurt am Main und des Frankfurter Technologiezentrums [:Medien] an der Fachhochschule Frankfurt am Main - University of Applied Sciences.» Erster Zwischenbericht über die 4. Projektlaufzeit von September 2011-August 2014. http://www.fraline.de/ images/stories/fraLineData/publikationen/projektberichte/zwischenbericht_ fraLine4_12November.pdf [aufgerufen am 13.7.2013].

Eickelmann, Birgit und Renate Schulz-Zander. 2006. "Schulentwicklung mit digitalen Medien - nationale Entwicklungen und Perspektiven.» In Jahrbuch der Schulentwicklung: Daten, Beispiele und Perspektiven, hrsg. v. Wilfried Bos, Heinz Günter Holtappels, Klaus Klemm, Hermann Pfeiffer, Hans-Günter Rolff u. Renate Schulz-Zander, 277-309. Weinheim u. München: Juventa.

Eimeren, Birgit van und Beate Frees. 2012. «76 Prozent der Deutschen online neue Nutzungssituation durch mobile Endgeräte. Ergebnisse der ARD/ZDFOnlinestudie 2012.» Media Perspektiven 7-8: 362-379. Online unter: www.media -perspektiven.de/uploads/tx_mppublications/0708-2012_Eimeren_Frees_01.pdf.

Herzig, Bardo und Silke Grafe. 2007. Digitale Medien in der Schule - Standortbestimmung und Handlungsempfehlungen für die Zukunft - Studie zur Nutzung digitaler Medien in allgemein bildenden Schulen in Deutschland. Bonn: Deutsche Telekom-AG.

Gysbers, Andre. 2008. Lehrer - Medien - Kompetenz. Berlin: Vistas.

Knaus, Thomas. 2009. Kommunigrafie - Eine empirische Studie zur Bedeutung von Text und Bild in der digitalen Kommunikation. München: kopaed.

Knaus, Thomas. 2011. "Digitale Medien - Eine Selbstverständlichkeit in Lehre und Unterricht? Analysen aus konstruktivistischer Perspektive.» In fraMediale - digitale Medien in Bildungseinrichtungen, hrsg. v. Thomas Knaus u. Olga Engel, 23-45. München: kopaed.

Knaus, Thomas und Dieter van Holst. 2012. «Was Lehrerinnen und Lehrer lernen (wollen) - Basics schulischer Medienbildung.» Computer + Unterricht 87: 11-13.

Klafki, Wolfgang. 1959. «Kategorale Bildung - Zur bildungstheoretischen Deutung der modernen Didaktik.» In Studien zur Bildungstheorie und Didaktik, 25-45. Weinheim: Beltz. 
Klafki, Wolfgang. 1991. Neue Studien zur Bildungstheorie und Didaktik - Zeitgemässe Allgemeinbildung und kritisch-konstruktive Didaktik. Weinheim: Beltz.

KMK-Kultusministerkonferenz. 2012. «Medienbildung in der Schule.» http://medien .bildung.hessen.de/dokumente/Beschluss-Empfehlung-Medienbildung -08-03-2012.pdf [aufgerufen am 16.7.2013].

Moser, Heinz. 2010. «Digital Divide in den Zeiten von Web 2.0 und Social Networks.» In Fokus Medienpädagogik - Aktuelle Forschungs- und Handlungsfeldern, hrsg. v. Petra Bauer, Hannah Hoffmann u. Kerstin Mayrberger, 135-149. München: kopaed.

Niesyto, Horst. 2009. «Digitale Medien, soziale Benachteiligung und soziale Distinktion.» MedienPädagogik 17 (Medien und soziokulturelle Unterschiede, 24.6.2006): www.medienpaed.com/17/\#niesyto0906 [aufgerufen am 16.7.2013].

Owston, Ronald. 2007. "Contextual factors that sustain innovative pedagogical practice using technology: an international study.» Journal of Educational Change 8 (1): 61-77.

Senkbeil, Martin. 2005. «Gleiche Chancen für alle? Befunde aus PISA 2003 zum Stellenwert der Schule beim Erwerb von Computerkenntnissen durch benachteiligte Jugendliche.» merz-medien + erziehung - Digitale Klüfte 49 (6): 97-109.

Sorge, Sarah. 2012 «Den Masterplan «Schule 2020» entwickeln und umsetzen.» Grusswort der Bildungsdezernentin Sarah Sorge im Rahmen der Eröffnung der fraMediale 2012 am 19. Sep. 2012. http://framediale.de/index.php/publikatio nen/65 [aufgerufen am 16.7.2013]

Thülen, Katharina. 2009. «Medienpädagogisch-technische Unterrichtsbegleitung. Niederschwellige Beratung und Begleitung bei der Nutzung neuer digitaler Medien im Unterricht.» In fraMediale - digitale Medien in Bildungseinrichtungen [Band 1], hrsg. v. Thomas Knaus u. Olga Engel, 137-149. München: kopaed.

Tulodziecki, Gerhard. 1998. "Entwicklung von Medienkompetenz als Erziehungsund Bildungsaufgabe.» Pädagogische Rundschau 52 (6): 693-709.

Tulodziecki, Gerhard. 2011. "Handeln und Lernen in einer von Medien mitgestalteten Welt - Konsequenzen für Erziehung und Bildung.» In Schulen in der digitalen Welt, hrsg. v. Carsten Albers, Johannes Magenheim u. Dorothee Meister, 43-64. Wiesbaden: VS Verlag für Sozialwissenschaften.

Wiedwald, Christian, Stefan Wilcke und Andreas Breiter. 2003. "Supportsituation der IT-Infrastruktur in den Schulen der Stadt Frankfurt am Main - Evaluation der Wirksamkeit und Akzeptanz des fraLine-IT-Schul-Service. Endbericht.» www.ifib.de/publikationsdateien/Fraline-Endbericht-020903.pdf [aufgerufen am 16.7.2013].

Wiedwald, Christian, Nicole Büsching und Andreas Breiter. 2007. «Pädagogische Medienentwicklungsplanung am Beispiel des Schulaufsichtsbezirks Frankfurt 
am Main - Zwischenbericht zur Mediennutzung in Schulen der Stadt Frankfurt am Main aus Sicht der Lehrkräfte.» www.ifib.de/publikationsdateien/Zwischen bericht-MEP-Frankfurt.pdf [aufgerufen am 16.7.2013].

Wiggenhorn, Gunhild und Oliver Vorndran. 2003. Computer in die Schule: Eine internationale Studie zu regionalen Implementationsstrategien. Gütersloh: Bertelsmann Stiftung. http://www.bertelsmann-stiftung.de/bst/de/media/xcms _bst_dms_17088_17089_2.pdf [aufgerufen am 16.7.2013]. 